\title{
Invariant measure and Lyapunov exponents for birational maps of $\mathrm{P}^{2}$
}

Jeffrey Diller

\begin{abstract}
In this paper we construct and study a natural invariant measure for a birational self-map of the complex projective plane. Our main hypothesis - that the birational map be "separating" - is a condition on the indeterminacy set of the map. We prove that the measure is mixing and that it has distinct Lyapunov exponents. Under a further hypothesis on the indeterminacy set we show that the measure is hyperbolic in the sense of Pesin theory. In this case, we also prove that saddle periodic points are dense in the support of the measure.
\end{abstract}

Mathematics Subject Classification (2000). Primary 32F50; Secondary 58F15, 58F23.

Keywords. Holomorphic dynamics, birational maps, Lyapunov exponents, invariant measures.

\section{Introduction}

In this paper and its predecessors [Dil1, Dil2] we develop an account of the dynamics of a birational map $f_{+}: \mathbf{P}^{2} \circlearrowleft$. The general idea, inspired by similar work [BS], [Bri], [FS1] in multi-variable complex dynamics, is to combine techniques from pluripotential and smooth ergodic theory to construct and then study several measure theoretic objects naturally associated with $f_{+}$. The difficulty in our context is the presence of the indeterminacy set $I^{+}$consisting of points at which $f_{+}$is ill-defined. Points of indeterminacy make potential theoretic constructions harder to accomplish and smooth ergodic theory more difficult to apply.

To proceed, let $\Theta$ be the Fubini-Study Kähler form on $\mathbf{P}^{2}$ and $d>1$ be the algebraic degree of $f_{+}$-i.e. the degree of the polynomials that define $f$ in homogeneous coordinates. We showed in [Dil1] that there exist positive closed $(1,1)$ currents

$$
\mu^{+}=\lim _{n \rightarrow \infty} \frac{1}{d^{n}} f_{+}^{n *} \Theta, \quad \mu^{-}=\lim _{n \rightarrow \infty} \frac{1}{d^{n}} f_{-}^{n *} \Theta
$$

associated with a birational map $f_{+}$and its inverse $f_{-}$, provided that $\operatorname{deg} f_{+}^{n}=d^{n}$ for all $n \geq 0$. Here we consider the measure $\mu=\mu^{+} \wedge \mu^{-}$. It is important

\footnotetext{
This research was partially supported by National Science Foundation grant \#DMS98-96370.
} 
to stress that the currents $\mu^{+}$and $\mu^{-}$are quite singular, especially at points of indeterminacy. In order to finesse the "multiplication" of $\mu^{+}$by $\mu^{-}$, we require that our birational maps be separating. That is, if $\mathcal{I}^{+}=\overline{\bigcup_{n>0} f_{-}^{n}\left(I^{+}\right)}$is the closure of the backward orbit of the indeterminacy set of $f_{+}$, and $\mathcal{I}^{-}$is the corresponding set for $f_{-}$, then we insist that $\mathcal{I}^{+} \cap \mathcal{I}^{-}=\emptyset$.

The currents $\mu^{+}$and $\mu^{-}$have the invariance properties $f_{+}^{*} \mu^{+}=d \cdot \mu^{+}, f_{-}^{*} \mu^{-}=$ $d \cdot \mu^{-}$. We show here that

Theorem 1.1. The measure $\mu$ is $f_{+}$-invariant.

The proof of this theorem is less immediate than one might hope. The key point is to show that $\mu$ attaches no mass to points of indeterminacy and, more generally, to the critical set of $f_{+}$. After establishing invariance, we adapt a proof of Bedford and Smillie [BS] to show that

Theorem 1.2. $\mu$ is mixing with respect to $f_{+}$.

Since mixing implies ergodicity, and the extended indeterminacy sets $\mathcal{I}^{+}$and $\mathcal{I}^{-}$are essentially invariant, this theorem has the consequence that at least one of the sets $\mathcal{I}^{+}$or $\mathcal{I}^{-}$is $\mu$-negligible.

The central results in this paper concern the Lyapunov exponents of $f_{+}$. Suppose for a moment that we are in the more general situation of a measurable, invertible and a.e. differentiable map $h: X \circlearrowleft$ of a compact two dimensional manifold and that $\nu$ is a probability measure that is ergodic with respect to $h$. Then under the hypothesis that the the derivatives of $h$ and $h^{-1}$ are log integrable with respect to $\nu$, Oseledec's Theorem guarantees the existence of two real numbers $\chi^{-}, \chi^{+}$that describe the growth rates of typical vectors under backward and forward iteration. That is, for $\nu$ a.e. point $p \in X$ and a generic vector $\mathbf{v} \in T_{p} X$, we have

$$
\lim _{n \rightarrow \infty} \frac{1}{n} \log \left\|h_{*}^{n} v\right\|=\chi^{+},
$$

and similarly for $h^{-1}$ and $\chi^{-}$. Oseledec's Theorem applies, in particular, if $h$ is an outright diffeomorphism. Here, we prove

Theorem 1.3. If $f_{+}: \mathbf{P}^{2} \circlearrowleft$ is birational and separating, then $\log ^{+}\left\|D f_{+}\right\|$is $\mu$ integrable. In particular, $f_{+}$satisfies the hypothesis of Oseledec's Theorem.

By $\log ^{+}(\cdot)$, we mean $\max \{\log (\cdot), 0\}$. An important ingredient in the proof of Theorem 1.3 is a rather technical result (Theorem 5.3) concerning regularity of local potentials for $\mu^{+}$. If $f_{+}$were holomorphic (i.e. $I^{+}=\emptyset$ ) instead of birational, the analogue for our regularity result would be that local potentials for $\mu^{+}$are Hölder continuous.

Lyapunov exponents are most meaningful when they are non-zero. The final result of this paper gives a simple criterion sufficient to guarantee that Lyapunov 
exponents of a separating birational map are non-zero.

Theorem 1.4. If $f_{+}: \mathbf{P}^{2} \circlearrowleft$ is a degree $d>1$ separating birational map and $\mu\left(\mathcal{I}^{+}\right)=0$, then $\chi^{+} \geq \log d / 4$.

Combined with the remark following Theorem 1.2, this implies that at least one of the Lyapunov exponents of a separating birational map is non-zero. Results similar to Theorem 1.4 have been obtained by Bedford and Smillie [BS] for polynomial diffeomorphisms of $\mathbf{C}^{2}$ and by Briend and Duval [Bri], [BD] for holomorphic maps of $\mathbf{P}^{n}$. The nearest precedent for the proof of Theorem 1.4 that we give here is Briend's thesis [Bri]. In particular, we appropriate his use of Lyapunov charts and coverings by balls of small $\mu$ mass.

If $\mu\left(\mathcal{I}^{+}\right)=\mu\left(\mathcal{I}^{-}\right)=0$, then Theorem 1.4 tells us that $\mu$ is (non-uniformly) hyperbolic - i.e. neither Lyapunov exponent vanishes. In this case, we adapt a standard proof of the closing lemma for uniformly hyperbolic maps to show

Theorem 1.5. If $f: \mathbf{P}^{2} \circlearrowleft$ is a degree $d>1$ separating birational map such that $\mu\left(\mathcal{I}^{+}\right)=\mu\left(\mathcal{I}^{-}\right)=0$, then $\operatorname{supp} \mu$ lies in the closure of the saddle periodic points of $f$.

We remark that it is not difficult to produce examples of separating birational maps. Any polynomial diffeomorphism of $\mathbf{C}^{2}$ extends to $\mathbf{P}^{2}$ as a birational map for which $\mathcal{I}^{+}$and $\mathcal{I}^{-}$are single (distinct) points. Hence a polynomial diffeomorphism of $\mathbf{C}^{2}$ is separating. Such a map remains separating if one pre- or post-composes with an automorphism of $\mathbf{P}^{2}$ close to the identity. More generally, if the $f_{-}$-orbit of each point in $I^{+}$converges to an $f_{-}$-attracting cycle, then $f_{+}$is separating and $\mu\left(\mathcal{I}^{+}\right)=0$. We refer the reader to the final section of [Dil1] for more specific examples.

This paper is organized as follows.

- Section 2 provides the necessary background on birational maps and pluripotential theory.

- Section 3 introduces the measure $\mu$ and contains the proof of Theorem 1.1. Many thanks to Eric Bedford for helping us with the pluripotential theory in this section.

- Section 4 contains the proof Theorem 1.2.

- Section 5 contains the proof of Theorem 1.3, including the regularity result for local potentials for $\mu^{+}$.

- Section 6 reviews the pertinent facts about Lyapunov exponents and Lyapunov charts. In particular, it states Oseledec's Theorem (suitably tailored to the present context).

- Section 7 contains the proof of Theorem 1.4.

- Section 8 contains the proof Theorem 1.5 


\section{Birational maps and pluripotential theory: background}

Let $\pi: \mathbf{C}^{3} \backslash\{\mathbf{0}\} \rightarrow \mathbf{P}^{2}$ denote the usual projection sending lines through $\mathbf{0}$ to points. Where a metric is implied but not specified in what follows, we assume the Euclidean metric on $\mathbf{C}^{3}$ and the Fubini-Study metric on $\mathbf{P}^{2}$. Recall that any homogeneous polynomial map $\tilde{f}: \mathbf{C}^{3} \rightarrow \mathbf{C}^{3}$ naturally induces a map $f: \mathbf{P}^{2} \rightarrow$ $\mathbf{P}^{2}$ satisfying $\pi \circ \tilde{f}=f \circ \pi$. Suppose that the coordinates of $\tilde{f}$ have no nonconstant common factors. Then we refer to the induced map $f$ as a rational map of (algebraic) degree $d \stackrel{\text { def }}{=} \operatorname{deg} \tilde{f}$. If $\tilde{f}(\tilde{p})=\mathbf{0}$ for some $\tilde{p} \neq \mathbf{0}$, then $f(\pi(\tilde{p}))$ cannot be defined continuously. We refer to $\pi(\tilde{p})$ as a point of indeterminacy for $f$ and denote the set of all such points by $I$. It is not hard to show that $I$ is always finite.

Throughout this paper, we will let $f_{+}: \mathbf{P}^{2} \circlearrowleft$ denote a birational map of algebraic degree $d>1$. That $f_{+}$is birational means that there exists an algebraic curve $V$ and another rational map $f_{-}: \mathbf{P}^{2} \rightarrow \mathbf{P}^{2}$ such that $f_{+} \circ f_{-}=f_{-} \circ f_{+}=$id on $\mathbf{P}^{2} \backslash V$. It turns out that the degree of $f_{-}$is also $d$.

We will distinguish objects corresponding to $f_{+}$from those corresponding to $f_{-}$using + and - sub/superscripts. For instance, we denote the critical set of $f_{+}$ by $\mathcal{C}^{+}$and remark that this set is an algebraic curve of degree $3 d-3$ counting multiplicity.

Proposition 2.1. The following statements are true for any birational map $f_{+}$: $\mathbf{P}^{2} \rightarrow \mathbf{P}^{2}$.

(1) $I^{+} \subset \mathcal{C}^{+}$, and every irreducible component of $\mathcal{C}^{+}$contains a point of $I^{+}$.

(2) Given any irreducible curve $V \subset \mathcal{C}^{+}, f_{+}(V)$ is a single point in $I^{-}$; likewise, given any $p^{-} \in I^{-}, f_{+}^{-1}\left(p^{-}\right)$is a component of $\mathcal{C}^{+}$.

(3) $f_{+}: \mathbf{P}^{2} \backslash \mathcal{C}^{+} \rightarrow \mathbf{P}^{2} \backslash \mathcal{C}^{-}$is a biholomorphism.

In particular, our assumption that $d>1$ implies that the critical sets $\mathcal{C}^{+}, \mathcal{C}^{-}$ and indeterminacy sets $I^{+}, I^{-}$are always non-empty. Proofs of Proposition 2.1 and of several of the following results can be found in [Dil1], [Dil2]. It is interesting to note that the algebraic degrees of $f_{+}^{n}$ do not necessarily grow as one would expect them to (i.e. one might guess that $\operatorname{deg} f_{+}^{n}=d^{n}=\operatorname{deg} \tilde{f}_{+}^{n}$ ). We require an extra hypothesis on $I^{+}$and $I^{-}$to guarantee predictable degree growth.

Proposition 2.2. The following statements are equivalent for a birational map $f_{+}: \mathbf{P}^{2} \rightarrow \mathbf{P}^{2}$ with inverse $f_{-}$:

(1) $\operatorname{deg}\left(f_{+}^{n}\right)=d^{n}$ for all $n$;

(2) $I^{+} \cap f_{+}^{n}\left(I^{-}\right)=\emptyset$ for all $n$.

(3) $f_{-}^{n}\left(I^{+}\right) \cap f_{+}^{m}\left(I^{-}\right)=\emptyset$ for all $n, m \geq 0$;

Following Sibony [Sib], we call maps satisfying any of these equivalent conditions algebraically stable. We will assume throughout this paper that all our birational maps belong to this category (in fact, shortly, we require something 
stronger). An important dynamical consequence of this assumption is the existence of a so-called Green's function $\tilde{G}^{+}$for $f_{+}$. The following theorem was proved in [Dil1] and in a very different fashion in [Fav]. However, Sibony [Sib] has given a quite simple proof that holds for general rational maps.

Theorem 2.3. Let $\tilde{f}_{+}$be a homogeneous representative for $f_{+}$. Then the limit

$$
\tilde{G}^{+}(\tilde{p})=\lim _{n \rightarrow \infty} \frac{1}{d^{n}} \log \left\|\tilde{f}_{+}^{n}(\tilde{p})\right\|
$$

exists pointwise and in $L_{\text {loc }}^{1}$. The function $\tilde{G}^{+}$is plurisubharmonic and satisfies

(1) $\tilde{G}^{+}(\lambda \tilde{p})=\tilde{G}^{+}(\tilde{p})+\log |\lambda|$ for every $\lambda \in \mathbf{C}$;

(2) $\tilde{G}^{+} \circ \tilde{f}_{+}=d \cdot \tilde{f}_{+}$.

The Green's function $\tilde{G}^{+}$is defined on $\mathbf{C}^{3}$ rather than on $\mathbf{P}^{2}$ and determined only up to an additive constant (depending on the choice of $\tilde{f}_{+}$). However, $d d^{c} \tilde{G}^{+}$ is a positive closed current independent of the additive constant, and it induces a positive closed current $\mu^{+}$on $\mathbf{P}^{2}$ as follows. Let $U \subset \mathbf{P}^{2}$ be open and $\sigma: U \rightarrow \mathbf{C}^{3}$ be a section of $\pi$. Then $\left.\mu^{+}\right|_{U}=d d^{c}\left(\tilde{G}^{+} \circ \sigma\right)$. It is quite natural to define $\left.f_{+}^{*} \mu^{+}\right|_{U}=$ $d d^{c}\left(\tilde{G}^{+} \circ \tilde{f}_{+} \circ \sigma\right)$, in which case it follows immediately that $f_{+}^{*} \mu^{+}=d \cdot \mu^{+}$. An additional fact about $\mu^{+}$(see [FS1]) that we will need is that $\mu^{+}$concentrates no mass on any algebraic curve.

We remark that any plurisubharmonic function $\tilde{u}: \mathbf{C}^{3} \rightarrow \mathbf{R} \cup\{-\infty\}$ satisfying $\tilde{u}(\lambda \tilde{p})=\tilde{u}(\tilde{p})+c \log |\lambda|$ for some $c>0$ induces a positive closed $(1,1)$ current $\nu$ on $\mathbf{P}^{2}$ in the same way that $\tilde{G}^{+}$does. It is not hard to see that $\langle\nu, \Theta\rangle=c$, where $\Theta$ is the Kähler form for the Fubini-Study metric on $\mathbf{P}^{2}$ appropriately normalized. Fornæss and Sibony [FS1] showed that every positive closed $(1,1)$ current on $\mathbf{P}^{2}$ is induced by a homogeneous potential $\tilde{u}$. In particular $\Theta$ is induced by $\log \|\tilde{p}\|$, so the first conclusion in Theorem 2.3 translates to the statement that

$$
\mu^{+}=\lim _{n \rightarrow \infty} \frac{1}{d^{n}} f_{+}^{n *} \Theta .
$$

In fact, $\mu^{+}$attracts a great many positive closed $(1,1)$ currents under pullback.

Theorem 2.4. Let $T$ be a closed $(1,1)$ current on $\mathbf{P}^{2}$. Suppose that $T$ has a bounded local potential (i.e. $T=d d^{c} u$, where $\|u\|_{\infty}<\infty$ ) on a neighborhood of each superattracting periodic point (if any) of $f_{+}$. Then

$$
\lim _{n \rightarrow \infty} \frac{1}{d^{n}} f_{+}^{n *} T=\mu^{+}
$$

The convergence takes place in the weak topology and is uniform among all $T$ whose support excludes a fixed neighborhood of all superattracting cycles.

We showed in [Dil2] that this theorem can be extended to certain 'truncated' currents. In order to state the more general theorem, we define the extended 
indeterminacy set of $f_{+}$to be the closure of the backward orbit of $I^{+}$- that is

$$
\mathcal{I}^{+}=\overline{\bigcup_{n=0}^{\infty} f_{-}^{n}\left(I^{+}\right)} \text {. }
$$

We also recall the mass norm of a positive current $T$ on an open set $U$ :

$$
\mathrm{M}_{U}[T]=\sup \left\{\langle T, \varphi\rangle: \operatorname{supp} \varphi \subset U,\|\varphi\|_{\infty} \leq 1\right\} .
$$

Of course convergence in mass norm is much stronger than convergence in the usual weak topology on currents. A slightly weakened version of Theorem 3.4 in [Dil2] goes as follows.

Theorem 2.5. Let $T$ be a positive closed $(1,1)$ current on $\mathbf{P}^{2}$ and $\psi: \mathbf{P}^{2} \rightarrow \mathbf{C}$ be a smooth function. Suppose that local potentials for $T$ are continuous on a neighborhood of $\mathcal{I}^{+} \cup \operatorname{supp} \psi$. Then

$$
\lim _{n \rightarrow \infty} \frac{1}{d^{n}} f_{-*}^{n}(\psi T)=\left(\int_{\mathbf{P}^{2}} \psi T \wedge \mu^{-}\right) \mu^{+} .
$$

Convergence takes place in the weak topology and is consistent with differentiation in that the sequences

$$
\frac{1}{d^{n}} \partial f_{-*}^{n}(\psi T), \quad \frac{1}{d^{n}} d d^{c} f_{-*}^{n}(\psi T)
$$

both tend to zero in the mass norm on $\mathbf{P}^{2}$.

Two points in the statement of this theorem merit explanation. The first is that the right side of the first equation implies that we can reasonably define the product $T \wedge \mu^{-}$as a measure. This is not obvious, but since the next section is devoted to a similar issue, we defer further discussion of wedge products of positive currents until then. The second point to explain is the use of $f_{-*}^{n}$ rather than $f_{+}^{n *}$ in both equations. While these notations are interchangeable in the setting of diffeomorphisms, we do not intend them to be so here. As we have already noted, it makes sense to pull back positive closed currents by pulling back their potentials. For present purposes, it suffices to take

$$
f_{-*}^{n}(\psi T)=\lim _{j \rightarrow \infty} f_{-*}^{n}\left(\chi_{j} \psi T\right)=\lim _{j \rightarrow \infty}\left(\chi_{j} \circ f_{+}^{n}\right) f_{-*}^{n}(\psi T)
$$

where $\chi_{j}: \mathbf{P}^{2} \rightarrow[0,1]$ is any sequence of smooth functions satisfying

- $\chi_{j}$ vanishes on a neighborhood of the critical set $\mathcal{C}_{n}^{+}$of $f_{-}^{n}$;

- $\chi_{j} \equiv 1$ on a set $K_{j}$ that increases to $\mathbf{P}^{2} \backslash \mathcal{C}_{n}^{+}$as $j$ goes to $\infty$.

More detailed discussion of the relationship between $f_{+}^{*}$ and $f_{-*}$ acting on positive currents occurs in [Dil2]. Suffice it to note here that if $\psi \equiv 1$ is trivial, then $f_{+}^{n *} T \geq f_{-*}^{n} T$. The case where $T$ is the current of integration over $\mathcal{C}_{n}^{-}$provides an example where the inequality is strict. 


\section{Invariant measure}

A formal construction suggests that pluripotential theory ought to yield an invariant measure for algebraically stable birational maps. Since $f_{+}$is algebraically stable if and only if $f_{-}$is, we can apply Theorem 2.3 to construct currents $\mu^{+}$and $\mu^{-}$associated with $f_{+}$and $f_{-}$, respectively. Then we set $\mu=\mu^{+} \wedge \mu^{-}$. It seems reasonable to expect that

$$
f_{+*} \mu=f_{+*} \mu^{+} \wedge f_{+*} \mu^{-}=\frac{\mu^{+}}{d} \wedge d \mu^{-}=\mu^{+} \wedge \mu^{-}=\mu .
$$

However, for the same reason that one cannot always multiply a pair of distributions together, it is not generally possible to form the wedge product of two currents. Furthermore, even if one can make sense of the wedge product, it remains to determine whether pushforward by $f_{+}$will distribute across the product as is assumed in (1). Our goal in this section is to address these difficulties and show that with a stronger hypothesis on $f_{+}$, the construction of an invariant measure from $\mu^{+}$and $\mu^{-}$succeeds.

Bedford and Taylor (see $[\mathrm{BT}]$ ) originated an integration by parts method for taking the wedge product of positive closed currents with locally bounded potentials. If $W \subset \mathbf{C}^{2}$ is open, $u: W \rightarrow \mathbf{R}$ is locally bounded and plurisubharmonic, and $T$ is a positive closed $(1,1)$ current on $W$, then the action of $d d^{c} u \wedge T$ on a test function $\varphi$ is given by

$$
\left\langle d d^{c} u \wedge T, \varphi\right\rangle=\left\langle T, u d d^{c} \varphi\right\rangle .
$$

It turns out that this defines $d d^{c} u \wedge T$ as a positive measure. This can be seen from the following theorem of Bedford and Taylor (see $[\mathrm{BT}]$ ).

Theorem 3.1. Suppose that $u_{j}, v_{j}: W \rightarrow \mathbf{R}$ are decreasing sequences of smooth plurisubharmonic functions converging pointwise to locally bounded plurisubharmonic functions $u, v$. Then

$$
\lim _{j \rightarrow \infty} d d^{c} u_{j} \wedge d d^{c} v_{j}=d d^{c} u \wedge d d^{c} v
$$

weakly.

Though examples indicate that the integration by parts construction cannot be used to defined the wedge product of arbitrary positive closed currents, one need not restrict oneself to positive closed currents with locally bounded potentials. Indeed, Fornæss and Sibony [FS2] have shown that the integration by parts construction and Theorem 3.1 succeed when the unboundedness loci of $u$ and $v$ do not coincide too much. The precise condition they discovered is as follows. Let $M_{u}$ denote the smallest closed set such that $p \notin M_{u}$ implies that $u_{j}$ is bounded on a neighborhood of $p$. Let $M_{v}$ be the corresponding set for $v$. Then the wedge product $d d^{c} u \wedge d d^{c} v$ is admissible provided that $M_{u} \cap M_{v}$ lies in the pseudoconvex 
envelope of its complement in $W$. In particular, things go well if at any point in $W$, at least one of the functions $u$ or $v$ is locally bounded.

Definition 3.2. We say that a birational map $f_{+}: \mathbf{P}^{2} \rightarrow \mathbf{P}^{2}$ is separating if $\mathcal{I}^{+} \cap \mathcal{I}^{-}=\emptyset$.

In particular, $f_{+}$is algebraically stable if it is separating. The following theorem is proved (in greater generality) in [Dil1].

Theorem 3.3. The Green function $\tilde{G}^{+}$for a separating birational map is continuous on $\pi^{-1}\left(\mathbf{P}^{2} \backslash \mathcal{I}^{+}\right)$.

In particular, local potentials for $\mu^{+}$are bounded near any point in $\mathbf{P}^{2} \backslash \mathcal{I}^{+}$. Clearly, $\left(\mathbf{P}^{2} \backslash \mathcal{I}^{+}\right) \cup\left(\mathbf{P}^{2} \backslash \mathcal{I}^{-}\right)=\mathbf{P}^{2}$ for a separating birational map, so we see from the discussion above that the wedge product $\mu=\mu^{+} \wedge \mu^{-}$is admissible for such a map. In order to show that $\mu$ is also invariant, we will need a couple of preliminary lemmas. We thank Eric Bedford for pointing these out to us and explaining their proofs.

Lemma 3.4. Suppose that $u$ and $v$ are plurisubharmonic functions defined on the unit polydisk $\Delta^{2}$, and that $u$ is continuous. Then $d d^{c} u \wedge d d^{c} v$ has no atoms.

Proof. It is enough to show that $d d^{c} u \wedge d d^{c} v$ attaches no mass to the origin. After subtracting off a constant, we can assume that $u(0,0)=0$ and set $\omega(r)=$ $\sup _{|x|,|y|<r}|u(x, y)|$. We choose a smooth compactly supported function $\psi: \Delta^{2} \rightarrow$ $[0,1]$ such that $\psi=1$ on $\Delta^{2} / 2$, and we set $\psi_{r}(x, y)=\psi(x / r, y / r)$ for $r>0$. Let $\theta=d d^{c}\|(x, y)\|^{2}$. Then since $d d^{c} u \wedge d d^{c} v$ is positive, we have

$$
\begin{aligned}
d d^{c} u \wedge d d^{c} v(\mathbf{0}) & \leq \liminf _{r \rightarrow 0} \int_{\Delta^{2}} \psi_{r} d d^{c} u \wedge d d^{c} v \\
& =\liminf _{r \rightarrow 0} \int_{\Delta^{2}} u d d^{c} \psi_{r} \wedge d d^{c} v \\
& =\liminf _{r \rightarrow 0}\left\|u d d^{c} \psi_{r}\right\|_{\infty} \mathrm{M}_{r \Delta^{2}}\left[d d^{c} v\right] \\
& \leq \liminf _{r \rightarrow 0} \frac{C \omega(r)}{r^{2}} \int_{r \Delta^{2}} \theta \wedge d d^{c} v
\end{aligned}
$$

But the last line is bounded above by $O(\omega(r))$ because of a consequence of Jensen's formula that we will use repeatedly in this paper (see e.g. [Dem], Consequence 4.4):

Fact 3.5. Suppose that $T$ is a positive closed $(1,1)$ current defined on a neighborhood of $\mathbf{0} \in \mathbf{C}^{2}$. Then

$$
\frac{1}{r^{2}} \int_{B_{\mathbf{0}}(r)} T \wedge \theta
$$


is an increasing function of $r$.

Since $\omega(r)$ tends to 0 with $r$, we are done.

Lemma 3.6. Suppose that $u$ and $v$ are plurisubharmonic functions on the unit polydisk $\Delta^{2}=\{|x|,|y|<1\}$. Assume that $u$ is continuous and that its restriction to the $x$ axis is harmonic. Assume that the restriction of $v$ to the $x$ axis is locally integrable (i.e. not identically $-\infty)$ ). Then $d d^{c} u \wedge d d^{c} v$ concentrates no mass on the $x$ axis.

Proof. Since the conclusion is true if and only if it holds for every open subset of the $x$-axis, we can assume without loss of generality that the restriction of $v$ to the $x$-axis is negative and (globally) integrable. By subtracting off $u(x, 0)$, we can assume that $u(x, y)$ vanishes on the $x$ axis. To prove the lemma, it will suffice to show that $d d^{c} u \wedge d d^{c} v$ places no mass on the disk $D=\{(x, 0):|x|<1 / 4\}$.

Let $\psi: \Delta \rightarrow[0,1]$ be a smooth, compactly supported function such that $\psi(z)=1$ if $|z| \leq 1 / 2$. Let $\psi_{r}(z)=\psi(z / r)$, and let

$$
\omega(r)=\sup \left\{u(x, y):|x|<\frac{1}{2},|y|<r\right\}
$$

Then

$$
\begin{aligned}
\int_{D} d d^{c} u \wedge d d^{c} v & =\lim _{r \rightarrow 0} \int_{\substack{|x|<1 / 4 \\
|y|<r}} d d^{c} u \wedge d d^{c} v \\
& \leq \lim _{r \rightarrow 0} \int_{\substack{|x|<1 / 2 \\
|y|<2 r}} \psi_{2 r}(y) \psi_{1 / 2}(x) d d^{c} u \wedge d d^{c} v \\
& =\lim _{r \rightarrow 0} \int_{\substack{|x|<1 / 2 \\
|y|<2 r}} u d d^{c}\left[\psi_{2 r}(y) \psi_{1 / 2}(x)\right] \wedge d d^{c} v
\end{aligned}
$$

We shall have to deal separately with each of the integrals that arises from expanding

$$
\begin{aligned}
d d^{c}\left[\psi_{2 r}(y) \psi_{1 / 2}(x)\right]= & \psi_{1 / 2}(x) d d^{c} \psi_{2 r}(y)+\psi_{2 r} d d^{c} \psi_{1 / 2}(x) \\
& +d \psi_{2 r}(y) \wedge d^{c} \psi_{1 / 2}(x)+d \psi_{1 / 2}(x) \wedge d^{c} \psi_{2 r}(y) .
\end{aligned}
$$

Consider the part of the integral corresponding to the first term in equation (3). In the following computation, we take advantage repeatedly of the fact that $\psi$ 
appears as a function of only one of the variables $x$ and $y$.

$$
\begin{aligned}
& \lim _{r \rightarrow 0} \int_{\substack{|x|<1 / 2 \\
|y|<2 r}} u \psi_{1 / 2}(x) d d^{c} \psi_{2 r}(y) \wedge d d^{c} v \\
& \leq \lim _{r \rightarrow 0} \frac{\omega(r)}{r^{2}}\left\|d d^{c} \psi\right\|_{\infty} \int_{\substack{|x|<1 / 2 \\
|y|<2 r}} d d^{c} v \wedge \frac{d y \wedge d \bar{y}}{2 i} \\
& \leq \lim _{r \rightarrow 0} \frac{C \omega(r)}{r^{2}} \int_{\substack{|x|<1 \\
|y|<4 r}} \psi_{4 r}(y) \psi(x) d d^{c} v \wedge \frac{d y \wedge d \bar{y}}{2 i} \\
&=\lim _{r \rightarrow 0} \frac{C \omega(r)}{r^{2}} \int_{\substack{|x|<1 \\
|y|<4 r}} v \psi_{4 r}(y) d d^{c} \psi(x) \wedge \frac{d y \wedge d \bar{y}}{2 i} \\
& \leq \lim _{r \rightarrow 0} \frac{C \omega(r)}{r^{2}} \int_{\substack{|x|<1 \\
|y|<4 r}}|v(x, y)| \frac{d y \wedge d \bar{y}}{2 i} \wedge \frac{d x \wedge d \bar{x}}{2 i} .
\end{aligned}
$$

But for almost every $x \in \Delta$, we have that

$$
\lim _{r \rightarrow 0} \frac{1}{16 \pi r^{2}} \int_{|y|<4 r}|v(x, y)| \frac{d y \wedge d \bar{y}}{2 i} \searrow|v(x, 0)| .
$$

Therefore, we can invoke the Lebesgue dominated convergence theorem and the fact that $\omega(r) \rightarrow 0$ with $r$ to conclude that the limit in (4) is zero. This takes care of the contribution to (2) from the first term on the right side of (3). The contribution from the second term can be handled in a similar fashion.

We can apply Schwarz's inequality to the contribution from the third term on the right side of $(3)$.

$$
\begin{aligned}
\lim _{r \rightarrow 0}\left|\int_{\substack{|x|<1 / 2 \\
|y|<2 r}} u d \psi_{2 r}(y) \wedge d^{c} \psi_{1 / 2}(x) \wedge d d^{c} v\right| \\
\leq \lim _{r \rightarrow 0}\left(\int_{\substack{|x|<1 / 2 \\
|y|<2 r}}|u| d \psi_{2 r}(y) \wedge d^{c} \psi_{2 r}(y) \wedge d d^{c} v\right)^{1 / 2} \\
\times\left(\int_{\substack{|x|<1 / 2 \\
|y|<2 r}}|u| d \psi_{1 / 2}(x) \wedge d^{c} \psi_{1 / 2}(x) \wedge d d^{c} v\right)^{1 / 2}
\end{aligned}
$$

By the same reasoning employed for the first term, we can show that each of the integrals in the last line behaves like $O(\omega(r))$ as $r$ tends to zero. In particular, the 
contribution to (2) from the third term in (3) vanishes. An identical argument shows that the contribution from the fourth term vanishes as well.

Theorem 3.7. The measure $\mu=\mu^{-} \wedge \mu^{+}$for a separating birational map has no atoms and puts no mass on $\mathcal{C}^{+}$or $\mathcal{C}^{-}$.

Proof. As we noted above, we can find a neighborhood $U=U(p)$ of any given point $p \in \mathbf{P}^{2}$ such that either $\tilde{G}^{+}$or $\tilde{G}^{-}$is continuous on $\pi^{-1}(U)$. Switching to local coordinates, we can assume that $U=\Delta^{2}$ is the unit polydisk, $p=(0,0)$ and $\sigma: \Delta^{2} \rightarrow \mathbf{C}^{3}$ is a holomorphic section. Since $\mu^{ \pm}=d d^{c} \tilde{G}^{ \pm} \circ \sigma$, Lemma 3.4 shows that $p$ is not an atom for $\mu$.

In particular, $\mu$ places no mass on $I^{+}$and no mass on any singular point of $\mathcal{C}^{-}$. To finish the proof, we need only show that $\mu$ places no mass on a neighborhood of each regular point of $\mathcal{C}^{+} \backslash I^{+}$and $\mathcal{C}^{-} \backslash I^{-}$. Take a regular point $p \in \mathcal{C}^{+} \backslash I^{+}$, for instance. Let $V$ be the irreducible component of $\mathcal{C}^{+}$containing $p$. Since $\tilde{G}^{-}$is continuous near $\pi^{-1}\left(I^{+}\right)$, we have from Proposition 2.1 that $\tilde{G}^{-}$is not identically equal to $-\infty$ on $V$. That is, local potentials for $\mu^{-}$are locally integrable on $V$. On the other hand, $f_{+}(V)$ is a point $p^{-} \in I^{-}$, and $\tilde{G}^{+}$is continuous in a neighborhood of $p^{-}$. We apply the formula $\tilde{G}^{+} \circ f_{+}=\left(\operatorname{deg} f_{+}\right) \tilde{G}^{+}$to conclude that $\tilde{G}^{+}$is continuous on a neighborhood of $\pi^{-1}\left(V \backslash I^{+}\right)$. Therefore, local potentials for $\mu^{+}$ are continuous on a neighborhood of $p$. Moreover, let $\sigma: U \rightarrow \mathbf{C}^{3}$ be a section defined on a neighborhood of $p$. Then the local potential $\tilde{G}^{+} \circ \sigma$ for $\mu^{+}$satisfies

$$
\tilde{G}^{+} \circ \sigma(q)=\frac{1}{\operatorname{deg} f_{+}} \tilde{G}^{+} \circ \tilde{f}_{+} \circ \sigma(q)=\frac{1}{\operatorname{deg} f_{+}}\left(\tilde{G}^{+}\left(\tilde{p}^{-}\right)\right)+\log |\lambda(q)|
$$

for all $q \in V \cap U$, some holomorphic function $\lambda: V \cap U \rightarrow \mathbf{C}^{*}$, and some $\tilde{p}^{-} \in \mathbf{C}^{3}$ (independent of $q$ ) such that $\pi\left(\tilde{p}^{-}\right)=p^{-}$. It follows that local potentials for $\mu^{+}$ are harmonic on $U \cap V$. We can take $U$ to be a small polydisk about $p$ such that $V \cap U$ is identified with the $x$-axis. Lemma 3.6 now applies to finish the proof.

Proof of Theorem 1.1. Recall from Proposition 2.1 that $f_{+}: \mathbf{P}^{2} \backslash \mathcal{C}^{+} \rightarrow \mathbf{P}^{2} \backslash \mathcal{C}^{-}$ is a biholomorphism. Therefore if $E \subset \mathbf{P}^{2} \backslash \mathcal{C}^{-}$, equation (1) holds rigorously. We need only consider further the case where $E \subset \mathcal{C}^{-}$. By the previous theorem, we have that $\mu(E)=0$. Furthermore, under any reasonable definition, $f_{+}^{-1}(E)$ will be a subset of $\mathcal{C}^{+}$. Hence, $\mu\left(f_{+}^{-1}(E)\right)=0$, too.

\section{Mixing}

A birational mapping $f_{+}: \mathbf{P}^{2} \rightarrow \mathbf{P}^{2}$ is said to be mixing with respect to an invariant measure $\mu$ if for any measurable subsets $A, B \subset \mathbf{P}^{2}$, we have

$$
\lim _{n \rightarrow \infty} \mu\left(f_{+}^{n}(A) \cap B\right)=\mu(A) \cap \mu(B) .
$$


Bedford and Smillie [BS] showed that polynomial diffeomorphisms of $\mathbf{P}^{2}$ are mixing with respect to the measure $\mu=\mu^{+} \wedge \mu^{-}$. We now generalize their result to separating birational maps. The main idea of the proof appears in [BS]. It is, however, somewhat more delicate to make this idea succeed at the present level of generality. As at the end of Section 2 we let $\mathcal{C}_{n}^{+}$denote the critical set of $f_{+}^{n}$.

Proof of Theorem 1.2. Since $\mu$ is a Borel measure, it is enough (see $[\mathrm{KH}]$ ) to show that for any two smooth functions $\psi, \varphi: \mathbf{P}^{2} \rightarrow \mathbf{C}$ we have

$$
\lim _{n \rightarrow \infty} \int_{\mathbf{P}^{2}} \varphi \cdot\left(\psi \circ f_{+}^{n}\right) d \mu=\left(\int_{\mathbf{P}^{2}} \varphi d \mu\right)\left(\int_{\mathbf{P}^{2}} \psi d \mu\right) .
$$

Even though $\psi \circ f_{+}^{n}$ might be discontinuous at points in $I_{n}^{+}$, the first integral makes sense because $\mu$ does not charge $\mathcal{C}_{n}^{+} \supset I_{n}^{+}$.

Clearly we lose no generality by assuming that $\psi$ and $\varphi$ take values only in the interval $[0,1]$ and that $\varphi$ is supported in a coordinate polydisk $D$. We can also assume that $D \cap \mathcal{I}^{-}=\emptyset$. To see this, note that because $f_{+}$is separating we can write $\varphi=\varphi^{+}+\varphi^{-}$where $\operatorname{supp} \varphi^{+} \cap \mathcal{I}^{-}$and $\operatorname{supp} \varphi^{-} \cap \mathcal{I}^{+}$are empty. Then by invariance of $\mu$, we can write

$$
\int_{\mathbf{P}^{2}} \varphi \cdot\left(\psi \circ f_{+}^{n}\right) d \mu=\int_{\mathbf{P}^{2}} \varphi^{+} \cdot\left(\psi \circ f_{+}^{n}\right) d \mu+\int_{\mathbf{P}^{2}} \psi \cdot\left(\varphi^{-} \circ f_{-}^{n}\right) d \mu .
$$

and deal with the first and second integrals separately. The arguments that follow address only the first integral, but those needed for the second integral are completely analogous.

We choose a local potential $g^{-}$for $\mu^{-}$on a neighborhood of $\bar{D}$ in such a way that $g^{-}$vanishes at every $z \in I_{n}^{+} \cap D$ (this can be arranged, since $I_{n}^{+}$is finite, by adding on an appropriate pluriharmonic function). We let

$$
\omega^{-}(r)=\max \left\{\left|g^{-}(z)\right|: z \in \overline{B_{I_{n}^{+}}(r)}\right\},
$$

and note that $\lim _{r \rightarrow 0} \omega^{-}(r)=0$ by Theorem 3.3 .

We choose smooth functions $\chi_{j}: \mathbf{P}^{2} \rightarrow[0,1]$ such that $\chi_{j}$ vanishes in a neighborhood of $\mathcal{C}_{n}^{+}$and that $\operatorname{supp}\left(1-\chi_{j}\right)$ decreases to $\mathcal{C}_{n}^{+}$as $j$ increases. For sufficiently small $r$ we choose smooth, compactly supported functions $\rho_{r}: D \rightarrow[0,1]$ as follows. Let $\rho: B_{0}(1) \rightarrow[0,1]$ be a smooth, compactly supported and radially symmetric function satisfying $\rho \equiv 1$ on $B_{0}(1 / 2)$. Using local coordinates on $D$, we then set

$$
\rho_{r}(z)=\sum_{w \in D \cap I_{n}^{+}} \rho\left(\frac{z-w}{r}\right) .
$$

In what follows we will repeatedly use the fact that if $T$ is a positive closed $(1,1)$ current on $\mathbf{P}^{2}$, and $\eta$ is a continuous function with absolute value less than one everywhere, then

$$
\left|\left\langle T, \eta d d^{c} \rho_{r}\right\rangle\right|,\left|\left\langle T, \eta d \rho_{r} \wedge d^{c} \rho_{r}\right\rangle\right| \leq \frac{C}{r^{2}}\left|\left\langle T, \rho_{2 r} \theta\right\rangle\right|
$$


where $\theta=d d^{c}\|z\|^{2}$ is the (local) Euclidean Kähler form on $D$, and $C$ depends on $\rho$ but not on $\eta$ or $r$.

To prove Theorem 4, we need to know that

\section{Lemma 4.1.}

$$
\int_{\mathbf{P}^{2}} \varphi \cdot\left(\psi \circ f_{+}^{n}\right) d \mu=\lim _{r \rightarrow 0}\left\langle\mu^{+}, g^{-}\left(1-\rho_{r}\right) d d^{c}\left[\varphi \cdot\left(\psi \circ f_{+}^{n}\right)\right]\right\rangle .
$$

In particular, the limit on the right side exists and is independent of $\rho$.

Assuming this lemma holds, the proof of the theorem proceeds as follows. We expand

$$
\begin{aligned}
d d^{c}\left[\varphi \cdot\left(\psi \circ f_{+}^{n}\right)\right]=\varphi d d^{c}\left(\psi \circ f_{+}^{n}\right)+ & d \varphi \wedge d^{c}\left(\psi \circ f_{+}^{n}\right) \\
& +d\left(\psi \circ f_{+}^{n}\right) \wedge d^{c} \varphi+\left(\psi \circ f_{+}^{n}\right) d d^{c} \varphi,
\end{aligned}
$$

and deal with the right side of (5) after distributing with respect to this decomposition. Taking advantage of invariance and the fact that $\mu^{+}$does not charge algebraic curves, we rewrite and bound

$$
\begin{aligned}
& \left|\left\langle\mu^{+}, g^{-}\left(1-\rho_{r}\right) \varphi d d^{c}\left(\psi \circ f_{+}^{n}\right)\right\rangle\right| \\
& =\lim _{j \rightarrow \infty} \frac{1}{d^{n}}\left|\left\langle f_{-*}^{n} \mu^{+}, \chi_{j} g^{-}\left(1-\rho_{r}\right) \varphi d d^{c}\left(\psi \circ f_{+}^{n}\right)\right\rangle\right| \\
& =\lim _{j \rightarrow \infty} \frac{1}{d^{n}}\left|\left\langle d d^{c} f_{-*}^{n}\left(\psi \mu^{+}\right), \chi_{j}\left(1-\rho_{r}\right) g^{-} \varphi\right\rangle\right| \\
& \leq \lim _{j \rightarrow \infty} \mathrm{M}\left[\frac{1}{d^{n}} d d^{c} f_{-*}^{n}\left(\psi \mu^{+}\right)\right]\left\|\chi_{j}\left(1-\rho_{r}\right) g^{-} \varphi\right\|_{\infty} \\
& \leq C \mathrm{M}\left[\frac{1}{d^{n}} d d^{c} f_{-*}^{n}\left(\psi \mu^{+}\right)\right]
\end{aligned}
$$

which, by Theorem 2.5, vanishes uniformly in $r$ as $n$ increases. The parts of (5) corresponding to the second and third terms on the right side of equation (6) vanish for similar reasons. Therefore, the only relevant term is the fourth one, which can be rewritten as

$$
\begin{aligned}
& \lim _{r \rightarrow 0}\left\langle\mu^{+}, g^{-}\left(1-\rho_{r}\right)\left(\psi \circ f_{+}^{n}\right) d d^{c} \varphi\right\rangle \\
& =\lim _{r \rightarrow 0} \lim _{j \rightarrow \infty} \frac{1}{d^{n}}\left\langle f_{+*}^{n} \mu^{+}, \chi_{j} g^{-}\left(1-\rho_{r}\right)\left(\psi \circ f_{+}^{n}\right) d d^{c} \varphi\right\rangle \\
& =\lim _{r \rightarrow 0} \lim _{j \rightarrow \infty} \frac{1}{d^{n}}\left\langle f_{+*}^{n}\left(\psi \mu^{+}\right), \chi_{j} g^{-}\left(1-\rho_{r}\right) d d^{c} \varphi\right\rangle \\
& =\frac{1}{d^{n}}\left\langle f_{+*}^{n}\left(\psi \mu^{+}\right), g^{-} d d^{c} \varphi\right\rangle,
\end{aligned}
$$


since $f_{+*}^{n}\left(\psi \mu^{+}\right)$does not charge $\mathcal{C}_{n}^{+}$or $I_{n}^{+}$. We conclude that

$$
\begin{aligned}
\lim _{n \rightarrow \infty} \int_{\mathbf{P}^{2}} \varphi \cdot\left(\psi \circ f_{+}^{n}\right) d \mu & =\lim _{n \rightarrow \infty} \frac{1}{d^{n}}\left\langle f_{+*}^{n}\left(\psi \mu^{+}\right), g^{-} d d^{c} \varphi\right\rangle \\
& =\left\langle\mu^{+} \wedge \mu^{-}, \psi\right\rangle\left\langle\mu^{+}, g^{-} d d^{c} \varphi\right\rangle \\
& =\langle\mu, \psi\rangle\left\langle\mu^{+} \wedge d d^{c} g^{-}, \varphi\right\rangle \\
& =\int \psi d \mu \cdot \int \varphi d \mu,
\end{aligned}
$$

as desired. The second equality follows from Theorem 2.5.

Proof of Lemma 4.1. By definition of $\mu$ and Theorem 3.7, we have

$$
\begin{aligned}
& \int_{\mathbf{P}^{2}} \varphi \cdot\left(\psi \circ f_{+}^{n}\right) d \mu= \lim _{r \rightarrow 0} \int_{\mathbf{P}^{2}}\left(1-\rho_{r}\right) \cdot \varphi \cdot\left(\psi \circ f_{+}^{n}\right) d \mu \\
&=\lim _{r \rightarrow 0}\left\langle\mu^{+}, g^{-} d d^{c}\left[\left(1-\rho_{r}\right) \cdot \varphi \cdot\left(\psi \circ f_{+}^{n}\right)\right]\right\rangle \\
&=\lim _{r \rightarrow 0}\left\langle\mu^{+}, g^{-}\left(1-\rho_{r}\right) d d^{c}\left[\varphi \cdot\left(\psi \circ f_{+}^{n}\right)\right]\right\rangle \\
& \\
&\left.\quad-\left\langle\mu^{+}, g^{-} \varphi \cdot\left(\psi \circ f_{+}^{n}\right) d d^{c} \rho_{r}\right)\right\rangle \\
& \\
&+\left\langle\mu^{+}, g^{-} d^{c}\left[\varphi \cdot\left(\psi \circ f_{+}^{n}\right)\right] \wedge d \rho_{r}\right\rangle \\
& \\
&+\left\langle\mu^{+}, g^{-} d^{c} \rho_{r} \wedge d\left[\varphi \cdot\left(\psi \circ f_{+}^{n}\right)\right]\right\rangle .
\end{aligned}
$$

Our task is to show that the last three terms vanish with $r$. The second term is most easily eliminated.

$$
\begin{aligned}
\lim _{r \rightarrow 0}\left|\left\langle\mu^{+}, g^{-} \varphi \cdot\left(\psi \circ f_{+}^{n}\right) d d^{c} \rho_{r}\right)\right\rangle \mid & \leq \lim _{r \rightarrow 0} C \omega^{-}(r) \frac{\left\langle\mu^{+}, \rho_{2 r} \theta\right\rangle}{r^{2}} \\
& \leq \lim _{r \rightarrow 0} C \omega^{-}(r)=0 .
\end{aligned}
$$

The third and fourth terms in (7) are equal, so we deal only with the third. We break this term up further.

$$
\begin{aligned}
\left|\left\langle\mu^{+}, g^{-} d^{c}\left(\varphi \cdot \psi \circ f_{+}^{n}\right) \wedge d \rho_{r}\right\rangle\right| \leq & \left|\left\langle\mu^{+}, g^{-}\left(\psi \circ f_{+}^{n}\right) d^{c} \varphi \wedge d \rho_{r}\right\rangle\right| \\
& +\left|\left\langle\mu^{+}, g^{-} \varphi d^{c}\left(\psi \circ f_{+}^{n}\right) \wedge d \rho_{r}\right\rangle\right| .
\end{aligned}
$$

To deal with the first term in this new decomposition, we apply Schwarz's inequality.

$$
\begin{aligned}
& \left|\left\langle\mu^{+}, g^{-}\left(\psi \circ f_{+}^{n}\right) d^{c} \varphi \wedge d \rho_{r}\right\rangle\right| \\
& \quad \leq\left|\left\langle\mu^{+},\left(g^{-}\left(\psi \circ f_{+}^{n}\right)\right)^{2} d \rho_{r} \wedge d^{c} \rho_{r}\right\rangle\right|^{1 / 2}\left|\left\langle\mu^{+}, d \varphi \wedge d^{c} \varphi\right\rangle\right|^{1 / 2} \\
& \quad \leq C \omega^{-}(r)\left(\frac{\left\langle\mu^{+}, \rho_{2 r} \theta\right\rangle}{r^{2}}\right)^{1 / 2} \leq C \omega^{-}(r)
\end{aligned}
$$

which tends to zero with $r$. It remains only to address the second term on the right side of (8). We apply Schwarz's inequality again and take advantage of the 
fact that $\mu^{+}$does not charge curves to compute

$$
\begin{aligned}
& \left|\left\langle\mu^{+}, g^{-} \varphi d^{c}\left(\psi \circ f_{+}^{n}\right) \wedge d \rho_{r}\right\rangle\right| \\
& \leq\left|\left\langle\mu^{+},\left(1-\rho_{r / 2}\right) d\left(\psi \circ f_{+}^{n}\right) \wedge d^{c}\left(\psi \circ f_{+}^{n}\right)\right\rangle\right|^{1 / 2}\left|\left\langle\mu^{+},\left(g^{-} \varphi\right)^{2} d \rho_{r} \wedge d^{c} \rho_{r}\right\rangle\right|^{1 / 2} \\
& \leq C \omega^{-}(r) \lim _{j \rightarrow \infty}\left|\left\langle\mu^{+}, \chi_{j}\left(1-\rho_{r / 2}\right) d\left(\psi \circ f_{+}^{n}\right) \wedge d^{c}\left(\psi \circ f_{+}^{n}\right)\right\rangle\right|^{1 / 2} \\
& =\frac{C \omega^{-}(r)}{d^{n / 2}} \lim _{j \rightarrow \infty}\left|\left\langle\mu^{+},\left(\chi_{j} \circ f_{-}^{n}\right)\left(1-\rho_{r / 2} \circ f_{-}^{n}\right) d \psi \wedge d^{c} \psi\right\rangle\right|^{1 / 2} \\
& \leq \frac{C \omega^{-}(r)}{d^{n / 2}} .
\end{aligned}
$$

Since the last quantity vanishes with $r$, we are done.

Corollary 4.2. If $f_{+}: \mathbf{P}^{2} \rightarrow \mathbf{P}^{2}$ is a separating birational map, then either $\operatorname{supp} \mu \subset \mathcal{I}^{+}$or $\mu\left(\mathcal{I}^{+}\right)=0$. In particular, either $\mu\left(\mathcal{I}^{+}\right)=0$ or $\mu\left(\mathcal{I}^{-}\right)=0$.

Proof. By the previous theorem $f_{+}$is ergodic with respect to $\mu$. By definition of $\mathcal{I}^{+}$, we have $f_{+}\left(\mathcal{I}^{+}\right)=\mathcal{I}^{+}$(modulo $I^{+}$, which has measure zero). Therefore, $\mu\left(\mathcal{I}^{+}\right)$is either zero or one. In the latter case, we conclude from the fact that $\mathcal{I}^{+}$ is closed that $\operatorname{supp} \mu \subset \mathcal{I}^{+}$. Finally, $\mathcal{I}^{+} \cap \mathcal{I}^{-}=\emptyset$ by hypothesis, so at least one of the two sets must have measure zero.

\section{Log integrability of the derivative}

In what follows (see the introduction to Section 6) it will be crucial to know that $\log ^{+}\left\|D f_{+}\right\|$and $\log ^{+}\left\|D f_{-}\right\|$are $\mu$ integrable functions. In order to establish integrability we will prove a strengthened continuity result for the Green's function $\tilde{G}^{+}$.

Fix a homogeneous map $\tilde{f}_{+}$inducing $f_{+}$. Given $p \in \mathbf{P}^{2}$ and $\tilde{p} \in \pi^{-1}(p)$, note that the quantity $\Gamma(p)=\log \frac{\left\|\tilde{f}_{+}(\tilde{p})\right\|}{\|\tilde{p}\|^{d}}$ depends on $p$ but not on $\tilde{p}$. Multiplying $\tilde{f}_{+}$ by a constant if necessary, we can assume $\Gamma \leq 0$ on all of $\mathbf{P}^{2}$. It is not difficult to verify that

$$
\tilde{G}^{+}(\tilde{p})=\log \|\tilde{p}\|+\sum_{n=0}^{\infty} \frac{\Gamma \circ f_{+}^{n}(p)}{d^{n}} .
$$

Further significance of $\Gamma$ stems from the following propositions proved in [Dil1].

Proposition 5.1. There is a constant $C$ such that for any $p, q \in \mathbf{P}^{2} \backslash I^{+}$.

$$
\operatorname{dist}\left(f_{+}(p), f_{+}(q)\right) \leq C e^{\max \{-\Gamma(p),-\Gamma(q)\}} \operatorname{dist}(p, q),
$$

Proposition 5.2. There are constants $C_{1}, C_{2}$ depending only on $f_{+}$such that

$$
C_{1}+C_{2} \log \operatorname{dist}\left(p, I^{+}\right) \leq \Gamma(p)
$$


In particular, $\Gamma$ is bounded below on any compact subset of $\mathbf{P}^{2} \backslash I^{+}$.

It follows (see Lemma 6.4 in [Dil1]) from Proposition 5.1 that there exists $C>0$ such that for any $p \in \mathbf{P}^{2}$ we have $\operatorname{dist}\left(f_{+}^{n}(p), \mathcal{I}^{+}\right) \geq C^{n} \operatorname{dist}\left(p, \mathcal{I}^{+}\right)$. It follows from Proposition 5.2 that

$$
\|d \Gamma(p)\|=\left\|d \frac{\|f(\tilde{p})\|}{\|\tilde{p}\|^{d}}\right\| e^{-\Gamma(p)} \leq C\left(\operatorname{dist}\left(p, I^{+}\right)\right)^{-k},
$$

where the first two d's denote exterior differentiation. We will make use of both these observations to prove the following upper bound on the modulus of continuity of the Green's function.

Theorem 5.3. Fix a compact set $K \subset \subset \mathbf{P}^{2} \backslash \mathcal{I}^{+}$. Then there exist constants $r, k>0$ such that

$$
\left|\tilde{G}^{+}\left(\frac{\tilde{p}}{\|\tilde{p}\|}\right)-\tilde{G}^{+}\left(\frac{\tilde{q}}{\|\tilde{q}\|}\right)\right| \leq e^{k \sqrt{|\log \operatorname{dist}(\pi(\tilde{p}), \pi(\tilde{q}))|}}
$$

for every $\tilde{p}, \tilde{q} \in \pi^{-1}(K)$ such that $\operatorname{dist}(\pi(\tilde{p}), \pi(\tilde{q})) \leq r$.

Remark 5.4. Note that if the square root were absent from the exponential in this theorem, then the conclusion would be that the Green's function is Hölder continuous.

Proof. Let $p=\pi(\tilde{p})$ and $q=\pi(\tilde{q})$ denote the corresponding points in $\mathbf{P}^{2}$. Set $r_{n}=\operatorname{dist}\left(f_{+}^{n}(p), f_{+}^{n}(q)\right)$ and $R_{n}=\operatorname{dist}\left(f_{+}^{n}(K), \mathcal{I}^{+}\right)$. By the first observation in the preceding paragraph, we see that

$$
R_{n} \geq C^{n} R_{0}
$$

for some constant $C=C\left(f_{+}\right)>0$. By (9) we have

$$
\left|\tilde{G}^{+}(\tilde{p})-\tilde{G}^{+}(\tilde{q})\right| \leq \sum_{n=0}^{\infty} \frac{\left|\Gamma \circ f_{+}^{n}(p)-\Gamma \circ f_{+}^{n}(q)\right|}{d^{n}}=S_{\text {init }}+S_{\text {tail }},
$$

where $S_{\text {init }}$ and $S_{\text {tail }}$ denote the first $n_{0}$ and remaining terms, respectively, in the sum. The value of $n_{0}$ will be determined in the course of our estimates below.

For terms in $S_{\text {tail }}$ we employ the crude upper bound

$$
\begin{aligned}
\left|\Gamma \circ f_{+}^{n}(p)-\Gamma \circ f_{+}^{n}(q)\right| & \leq \max \left\{-\Gamma \circ f_{+}^{n}(p),-\Gamma \circ f_{+}^{n}(q)\right\} \\
& \leq C_{1}+n C_{2},
\end{aligned}
$$

where $C_{1}, C_{2}>0$ are constants depending on $f_{+}$and $K$. The second inequality 
follows from Proposition 5.2 and equation (11). Hence,

$$
\begin{aligned}
S_{\text {tail }} & \leq \sum_{n=n_{0}}^{\infty} \frac{C_{1}+n C_{2}}{d^{n}} \\
& \leq \sum_{n_{0} \leq n \leq 10} \frac{C_{1}+n C_{2}}{d^{n}}+\sum_{n \geq \max \left\{n_{0}, 10\right\}}^{\infty} \frac{C_{1}+n C_{2}}{d^{n}} \\
& \leq \frac{C}{d^{n_{0}}}+\sum_{n \geq \max \left\{n_{0}, 10\right\}}^{\infty} \frac{C}{\lambda^{n_{0}}},
\end{aligned}
$$

where $\lambda=\frac{10}{11}$ and $d>1$. We conclude that

$$
S_{\text {tail }} \leq \frac{C}{\lambda^{n_{0}}}=C e^{-k n_{0}},
$$

where $C, k>0$ are constants depending only on $f_{+}$and $K$.

Lemma 5.5. Suppose that $r_{j}<R_{j}$ for all $j<n$. Then

$$
r_{n} \leq C^{n^{2}} r_{0}
$$

where $C>0$ depends only on $K$ and $f_{+}$. Moreover, there exists a constant $A=$ $A\left(f_{+}, K\right)$ such that $r_{j}<R_{j}$ for all $j<A \sqrt{\left|\log r_{0}\right|}$.

Proof. According to Proposition 5.1, we have

$$
r_{n} \leq \frac{C r_{n-1}}{R_{n-1}^{k}} \leq \cdots \leq \frac{C^{n} r_{0}}{\left(R_{n-1} \ldots R_{0}\right)^{k}} .
$$

But equation (11) then gives

$$
r_{n} \leq \frac{C_{1}^{n} r_{0}}{C_{2}^{n(n+1) / 2} R_{0}^{k n}} \leq C^{n^{2}} r_{0},
$$

as desired. The last part of the lemma follows inductively from the first via the estimate

$$
r_{j} \leq C^{j^{2}} r_{0} \leq B^{j} R_{0} \leq R_{j},
$$

where we assume without loss of generality that $C>1>B$.

We now return to the proof of Theorem 5.3, estimating $S_{\text {init }}$ under the assumption that $n_{0}$ is no larger than $A \sqrt{\left|\log r_{0}\right|}$. We also assume that $r_{0}<R_{0}$. It then follows from Lemma 5.5 that $r_{n}<R_{n}$ for all $n<n_{0}$. In particular, the geodesic segment $\ell$ joining $f_{+}^{n}(p)$ to $f_{+}^{n}(q)$ will avoid $I^{+}$by a distance of at least $R_{n} / 2$. Thus by Lemma 5.5 and equation (11),

$$
\left|\Gamma \circ f_{+}^{n}(p)-\Gamma \circ f_{+}^{n}(q)\right| \leq \max _{t \in \ell}\|d \Gamma(t)\| r_{n} \leq C r_{n}\left(\frac{2}{R_{n}}\right)^{k} \leq C^{n^{2}+1} r_{0},
$$


for some $C>1$ and all $n<n_{0}$. We conclude that

$$
S_{\text {init }}=\sum_{n=0}^{n_{0}-1} \frac{\left|\Gamma \circ f_{+}^{n}(p)-\Gamma \circ f_{+}^{n}(q)\right|}{d^{n}} \leq \sum_{n=0}^{n_{0}-1} \frac{C^{n^{2}+1} r_{0}}{d^{n}} \leq C^{n_{0}^{2}+1} r_{0} .
$$

Now suppose in addition that $r_{0}<1 / 2$. After possibly shrinking the constant $A$ in Lemma 5.5 and taking $n_{0}$ to be the least integer larger than $A \sqrt{\left|\log r_{0}\right|}$, we will have $S_{\text {init }}<C r_{0}^{1 / 2}$. From equation (13), we will further have $S_{t a i l}<e^{-B \sqrt{\left|\log r_{0}\right|}}$. For small $r_{0}$, the larger of these bounds is the one for $S_{\text {tail }}$. Since $r_{0}=\operatorname{dist}(p, q)$, we are done.

Remark 5.6. The constants $k$ and $r_{0}$ in Theorem 5.3 depend on $K$ (i.e. on the distance $R_{0}$ from $K$ to $\mathcal{I}^{+}$). It is entirely possible, though somewhat messier, to keep track of this dependence throughout the proof of the theorem and obtain estimates that are completely explicit in terms of $R_{0}$.

We are now ready for the

Proof of Theorem 1.3. It is enough to know that $\log ^{+}\left\|D f_{-}\right\|$is locally integrable near each point in $\mathbf{P}^{2}$. Near points $p \notin I^{+}, \log ^{+}\left\|D f_{-}\right\|$is continuous. Since $\mu$ is a Borel measure, local integrability near $p$ is automatic.

It remains to verify local integrability near each point $p$ in the finite set $I^{+}$. Without loss of generality, we work in local coordinates $z$ such that $p$ corresponds to $z=\mathbf{0}$. It follows from Proposition 5.1 that

$$
\log ^{+}\left\|D f_{-}(z)\right\| \leq A \log \frac{1}{\|z\|}+B
$$

Therefore, we need only show that $\log \|z\|$ is $\mu$ integrable in a ball $B_{\mathbf{0}}(1)$ of radius one about $\mathbf{0}$. We estimate this integral by dividing the ball into shells.

$$
\begin{aligned}
\int_{B_{\mathbf{0}}(1)} \log \frac{1}{\|z\|} \mu & =\sum_{j=0}^{\infty} \int_{\frac{1}{2^{j+1}}<\|z\|<\frac{1}{2^{j}}} \log \frac{1}{\|z\|} \mu \\
& \leq \sum_{j=0}^{\infty}(j+1)(\log 2) \mu\left(B_{\mathbf{0}}\left(2^{-j}\right)\right) .
\end{aligned}
$$

Let $\theta$ be the Euclidean Kähler form on $B_{\mathbf{0}}(2)$, and choose a cutoff function $\psi: B_{\mathbf{0}}(2) \rightarrow[0,1]$. That is, $\psi$ is smooth, radially symmetric, and compactly supported on $B_{\mathbf{0}}(2)$, and $\psi \equiv 1$ on $B_{\mathbf{0}}(1)$. We let $\psi_{j}(z)=\psi\left(2^{j} z\right)$, noting that the $C^{2}$ norm of $\psi_{j}$ is $4^{j}$ times that of $\psi$. Since $\theta$ is a strongly positive form (see [Kli]), we have a constant $C>0$ such that $\left|\left\langle T, \rho d d^{c} \psi_{j}\right\rangle\right|<C 4^{j}\langle T,|\rho| \theta\rangle$ for any positive closed $(1,1)$ current $T$ and any continuous function $\rho$.

The map $f_{+}$is separating, so we lose no generality by assuming that $\mathcal{I}^{+} \cap B_{\mathbf{0}}(2)$ is empty. Theorem 3.3 then implies that $\mu^{+}$has a continuous local potential on $B_{\mathbf{0}}(2)$. That is, we choose a section $\sigma: B_{\mathbf{0}}(2) \subset \mathbf{P}^{2} \rightarrow \mathbf{C}^{3} \backslash\{\mathbf{0}\}$ of the canonical 
projection $\pi$ and define the local potential $g^{+}(z)=\tilde{G}^{+} \circ \sigma(z)-\tilde{G}^{+} \circ \sigma(\mathbf{0})$. Again without losing generality, we assume that the $C^{1}$ norm of $\log \|\sigma\|$ is uniformly bounded. This combined with Theorem 5.3 gives us for each $\|z\|<r \leq 2$ that

$$
\begin{aligned}
\left|g^{+}(z)\right| & =\left|\tilde{G}^{+} \circ \sigma(z)-\tilde{G}^{+} \circ \sigma(0)\right| \\
& \leq\left|\tilde{G}^{+}\left(\frac{\sigma(z)}{\|\sigma(z)\|}\right)-\tilde{G}^{+}\left(\frac{\sigma(0)}{\|\sigma(0)\|}\right)\right|+\left|\log \frac{\|\sigma(z)\|}{\|\sigma(0)\|}\right| \\
& \leq e^{-k \sqrt{|\log r|}}+C r \leq e^{-k \sqrt{|\log r|}} .
\end{aligned}
$$

The first inequality follows from item 1 in Theorem 2.3. The integration by parts definition of $\mu$ allows us to compute

$$
\begin{aligned}
\mu\left(B_{\mathbf{0}}\left(2^{-j}\right)\right) & \leq \int_{B_{\mathbf{0}}(1)} \psi_{j} \mu^{+} \wedge \mu^{-} \\
& =\int_{B_{\mathbf{0}}(1)} g^{+} d d^{c} \psi_{j} \wedge \mu^{-} \\
& \leq C\left\|\psi_{j}\right\|_{C^{2}} \sup _{B_{\mathbf{0}}\left(2^{-j+1}\right)}\left|g^{+}\right| \int_{B_{\mathbf{0}}\left(2^{-j+1}\right)} \theta \wedge \mu^{-} \\
& \leq C e^{-k \sqrt{j}} \frac{1}{4^{-j}} \int_{B_{\mathbf{0}}\left(2^{-j+1}\right)} \theta \wedge \mu^{-} . \\
& \leq C e^{-k \sqrt{j}},
\end{aligned}
$$

for all $j>0$ (the last inequality comes from Fact 3.5). Inserting this estimate into (14), we obtain

$$
\int_{B_{\mathbf{0}}(1)} \log \frac{1}{\|z\|} \mu \leq \sum_{j=0}^{\infty} C j e^{-k \sqrt{j}} .
$$

One can see that the last sum converges by comparing its terms with $j^{-2}$ for $j$ large. This completes the proof.

Corollary 5.7. If $f_{+}$is separating, then the functions $\log ^{+}\left\|D f_{ \pm}^{-1}\right\|$ are also integrable.

Proof. Since $\mu$ is invariant and $D f_{+}^{-1} \circ f_{+}=D f_{-}$at $\mu$ almost every point, we have

$$
\int_{\mathbf{P}^{2}} \log ^{+}\left\|D f_{+}^{-1}\right\| \mu=\int_{\mathbf{P}^{2}} \log ^{+}\left\|D f_{+}^{-1} \circ f_{+}\right\| f_{-*} \mu=\int_{\mathbf{P}^{2}} \log ^{+}\left\|D f_{-}\right\| \mu<\infty .
$$

\section{Lyapunov exponents and Lyapunov charts}

Since the quantities $\log ^{+}\left\|D f_{+}^{ \pm 1}\right\|$ are $\mu$ integrable, we can apply the well-known (see the supplemental section in $[\mathrm{KH}]$ ) multiplicative ergodic theorem of Oseledec. 
Taking into account the fact that our invariant measure is mixing, we immediately deduce the following from Oseledec's Theorem.

Theorem 6.1. Suppose that $f_{+}: \mathbf{P}^{2} \rightarrow \mathbf{P}^{2}$ is a separating birational map. There exist numbers $\chi^{-} \leq \chi^{+}$such that at $\mu$ almost every point $p \in \mathbf{P}^{2}$

$$
\chi^{+}=\lim _{n \rightarrow \infty} \frac{1}{n} \log \left\|D f_{+}^{n}(p)\right\|, \quad \chi^{-}=-\lim _{n \rightarrow \infty} \frac{1}{n} \log \left\|D f_{-}^{n}(p)\right\| .
$$

If $\chi^{-}=\chi^{+}$, then

$$
\chi^{+}=\chi^{-}= \pm \lim _{n \rightarrow \infty} \frac{1}{n} \log \left\|D f_{ \pm}^{n}(p) \cdot v\right\|
$$

for almost every point $p \in \mathbf{P}^{2}$ and any non-zero vector $v \in \mathrm{T}_{p} \mathbf{P}^{2}$. If $\chi^{+}>\chi^{-}$, then there exists a measurably varying, $f_{+}$invariant decomposition $\mathrm{T}_{p} \mathbf{P}^{2}=\mathrm{E}^{s} \oplus \mathrm{E}^{u}$ at $\mu$ almost every point of $\mathbf{P}^{2}$. Moreover, if $v \in \mathrm{E}^{s} \backslash\{\mathbf{0}\}$, then

$$
\chi^{-}=\lim _{n \rightarrow \infty} \frac{1}{n} \log \left\|D f_{+}^{n}(p) \cdot v\right\|=-\lim _{n \rightarrow \infty} \frac{1}{n} \log \left\|D f_{-}^{n}(p) \cdot v\right\|
$$

and similarly for $v \in \mathrm{E}^{u} \backslash\{\mathbf{0}\}$ and $\chi^{+}$. Finally, the sine of the angle between $\mathrm{E}^{s}$ and $\mathrm{E}^{u}$ is "tempered" in the sense that

$$
\lim _{n \rightarrow \infty} \frac{1}{n} \log \sin \angle\left(\mathrm{E}_{f_{ \pm}^{n}(p)}^{s}, \mathrm{E}_{f_{ \pm}^{n}(p)}^{u}\right)=0
$$

at almost every point $p$.

The numbers $\chi^{+}$and $\chi^{-}$are called the Lyapunov exponents of $f_{+}$with respect to $\mu$. The theory of non-uniform hyperbolicity (see $[\mathrm{KH}]$ for an introduction and further references; I also learned a great deal from Briend's thesis [Bri] which contains a nice general exposition of these topics in a context similar to ours) was developed by Pesin and others in order to explore the way in which Lyapunov exponents influence the dynamics of a map. This theory begins with $([\mathrm{KH}]$, Theorem S.2.10) the fact that one can make a tempered, measurably varying choice of coordinates on tangent spaces $\mathrm{T}_{p}$ so that $D_{p} f_{+}$has Lyapunov block form. That is, in these coordinates $D_{p} f_{+}$acts, up to an error factor of $e^{\epsilon}$, like a diagonal matrix with entries of absolute value $e^{\chi^{+}}, e^{\chi^{-}}$. Moreover, in the sense specified by the following result ([KH], Theorem S.3.1), the infinitesimal choice of coordinates on $\mathrm{T}_{p}$ can be used to select advantageous local coordinates on a neighborhood of $p$.

Theorem 6.2. Suppose $f_{+}: \mathbf{P}^{2} \rightarrow \mathbf{P}^{2}$ is a separating birational map, and let $\chi^{+}, \chi^{-}, \mathrm{E}^{s}, \mathrm{E}^{u}$ be as above. For any $\epsilon>0$ there exists a set $\Lambda \subset \operatorname{supp} \mu$ of full measure, a tempered function $\delta: \Lambda \rightarrow(0,1]$, and a collection of holomorphic embeddings $\psi_{p}: B_{\mathbf{0}}(\delta(p)) \rightarrow \mathbf{P}^{2}$ such that

(1) $\psi_{p}(\mathbf{0})=p$;

(2) $\delta$ is $\epsilon$-slowly varying-i.e. $e^{-\epsilon}<\delta\left(f_{+}(p)\right) / \delta(p)<e^{\epsilon}$ for every $p \in \Lambda$; 
(3) There exists a constant $K>0$ and a measurable, $\epsilon$-slowly varying function $A: \Lambda \rightarrow \mathbf{R}^{+}$such that

$$
K^{-1} \operatorname{dist}\left(\psi_{p}(x), \psi_{p}(y)\right)<\|x-y\|<A(p) \operatorname{dist}\left(\psi_{p}(x), \psi_{p}(y)\right) .
$$

(4) If $f_{p}=\psi_{f_{+}(p)}^{-1} \circ f_{+} \circ \psi_{p}$, then $f_{p}$ and $D_{\mathbf{0}} f_{p}$ are $\epsilon$ close in the $C^{1}$ distance on $B_{\mathbf{0}}(\delta(p))$;

(5) $D_{0} f_{p}$ has Lyapunov block form.

We will call the maps $\psi_{p}$ Lyapunov charts and the points $p \in \Lambda$ regular. Since $\Lambda$ is of full measure, we can (and will) assume that $p \in \Lambda$ implies that $f^{n}(p) \in \Lambda$ for all $n \in \mathbf{Z}$. The proof of Theorem 6.2 is given for $C^{1+\alpha}$ diffeomorphisms in $[\mathrm{KH}]$, and it applies almost directly to the case of separating birational maps. The only additional technicality in our setting is that the $C^{2}$ norm of $f_{+}$becomes unbounded near points of indeterminacy. This affects the definition of $\delta$, and in particular, the conclusion that we can choose a slowly varying $\delta$. However, one can show that the $\log$ of the $C^{2}$ norm of $f_{+}$is integrable in the same way that we proved integrability of $\log \left\|D f_{+}\right\|$in the previous section. Further inspection of the proof in $[\mathrm{KH}]$ reveals that this integrability suffices to overcome the extra difficulty.

Corollary 6.3. The Lyapunov exponents for a separating birational map $f_{+}$satisfy $\chi^{-} \leq 0 \leq \chi^{+}$.

Proof. Suppose that both exponents are negative, and apply Theorem 6.2 with $\epsilon<<\left|\chi^{+}\right|$. Consider a Lyapunov chart $\psi_{p}: B_{\mathbf{0}}(\delta(p)) \rightarrow \mathbf{P}^{2}$ about a point $p \in \Lambda$. Then $f_{p}$ maps $B_{\mathbf{0}}(\delta(p))$ into $B_{\mathbf{0}}\left(\delta\left(f_{+}(p)\right)\right)$, contracting by a factor of at least $e^{\chi^{+}+\epsilon}$. Likewise $f_{f_{+}(p)}, f_{f_{+}^{2}(p)}$, etc, will further contract images of the ball by the same factor so that successive images will eventually decrease to $\{\mathbf{0}\}$. By item 3 . of Theorem 6.2 , we conclude that the radius of $f_{+}^{n}\left(\psi_{p} B_{\mathbf{0}}(\delta(p))\right.$ tends to zero as $n$ increases. In particular, iterates of $f_{+}$form a normal family in a neighborhood of $p$. This implies (see [Dill]) that $p \notin \operatorname{supp} \mu^{+}$, and therefore, that $p \notin \Lambda \subset \operatorname{supp} \mu$.

\section{Lower estimate for Lyapunov exponents}

The goal of this section is to prove Theorem 1.4. Before we start, we state an immediate consequence of Theorem 1.4 and Corollary 4.2.

Corollary 7.1. The measure $\mu$ associated with a separating birational map has at least one non-zero Lyapunov exponent. Moreover, either $\mu$ is a hyperbolic measure (i.e. neither exponent vanishes) or $\operatorname{supp} \mu \subset \mathcal{I}^{+} \cup \mathcal{I}^{-}$. 
For purposes of this section and the next, we fix some notation. Given an $\epsilon>0$, we apply Theorem 6.2 to $f_{+}$. Let $t$ be a number between 0 and 1 . Since the resulting Lyapunov charts vary measurably, we can find compact subsets $\Lambda_{t} \subset \Lambda$ of measure $1-t$ such that $\mu\left(\Lambda_{t}\right)>1-t$ and the Lyapunov charts vary continuously on $\Lambda_{t}$ in the $C^{1}$ topology. In particular, the function $\delta$ admits a positive lower bound $\delta_{t}$ on $\Lambda_{t}$ and the function $A$ admits an upper bound $A_{t}$.

In proving Theorem 1.4, we take $t=1 / 2$. The set $\mathcal{I}^{+}$is $\mu$ negligible by hypothesis and closed by definition, so we can certainly assume that $\Lambda_{1 / 2}$ is disjoint from $\mathcal{I}^{+}$. By shrinking $\delta$ if necessary, we can further arrange that $\operatorname{dist}\left(\Lambda_{1 / 2}, \mathcal{I}^{+}\right)>$ $2 K \delta_{1 / 2}$. This and Theorem 6.2 guarantees that the image $\psi_{p}\left(B_{0}\left(\delta_{1 / 2}\right)\right)$ of the Pesin chart about each $p \in \Lambda_{1 / 2}$ avoids $\mathcal{I}^{+}$by a positive distance independent of $p$. We proceed in a series of lemmas.

Lemma 7.2. There exists a constant $C$ and for each $p \in \Lambda_{1 / 2}$ a local potential $g_{p}^{+}$ for $\mu^{+}$on $\psi_{p}\left(B_{\mathbf{0}}\left(\delta_{1 / 2}\right)\right)$ such that $\left\|g_{p}^{+}\right\|_{\infty}<C$.

Proof. By construction of $\Lambda_{1 / 2}$ and Theorem 3.3, the Green's function $\tilde{G}^{+}$is uniformly continuous over the set $\bigcup_{p \in \Lambda_{1 / 2}} \psi_{p}\left(B_{\mathbf{0}}\left(\delta_{1 / 2}\right)\right)$. Fix $p \in \Lambda_{1 / 2}$ and choose a section $\sigma: \psi_{p}\left(B_{\mathbf{0}}\left(\delta_{1 / 2}\right)\right) \rightarrow \mathbf{C}^{3} \backslash\{\mathbf{0}\}$ such that $\|\sigma(p)\|=1$ and the image of $\sigma$ is contained in the complex hyperplane tangent to the unit sphere at $\sigma(p)$ - e.g. if $\sigma(p)=(0,0,1)$, then the first two components of $\sigma$ give affine coordinates on $\psi_{p}\left(B_{\mathbf{0}}\left(\delta_{1 / 2}\right)\right)$. Because the images of the Lyapunov charts are uniformly small, the image of $\sigma$ is also contained in a spherical shell $\{1 \leq\|\tilde{p}\| \leq C\}$ for some constant $C$ independent of $p$. The proof is concluded by taking $g_{p}^{+}=\tilde{G}^{+} \circ \sigma$.

Since $f_{+}$can be conjugated near any point in $\Lambda_{1 / 2}$ to within $\epsilon$ of a non-singular matrix, we see that $f_{+}^{n}\left(I^{-}\right) \cap \Lambda_{1 / 2}=\emptyset$ for every $n$. In particular, the sets $S_{n}=$ $f_{-}^{n}\left(\Lambda_{1 / 2}\right) \cap \Lambda_{1 / 2}$ are well-defined and compact for every $n \in \mathbf{N}$.

Lemma 7.3. There exist constants $C, N>0$ such that for every $n \geq N$ :

(1) $\mu\left(S_{n}\right)>1 / 8$;

(2) for every $r<\delta_{1 / 2}$ and any $n>N$, there exists points $p_{1}, \ldots, p_{k} \in S_{n}$ such that $k<C / r^{4}$ and $S_{n} \subset \bigcup_{j=1}^{k} \psi_{p_{j}}\left(B_{0}(r)\right)$.

Proof. The first assertion follows from the fact that $\mu$ is mixing for $f_{-}$. Given $r<\delta_{1 / 2}$ consider the collection of open sets of the form $\psi_{p}\left(B_{\mathbf{0}}(r)\right)$ where $p$ is any point in $S_{n}$. By Theorem 6.2, each of these open sets contains a round ball $B_{p}\left(r / A_{1 / 2}\right)$. Since $S_{n}$ is compact, we can choose finitely many points $p_{1}, \ldots, p_{k}$ so that the balls $B_{p}\left(r / 5 A_{1 / 2}\right)$ centered at these points cover $S_{n}$. Moreover, by a well-known argument (see the proof in 1.6 of [Ste]), we can discard some of these balls (i.e. re-index and shrink $k$ ) so that the remaining balls are mutually disjoint but that when we expand their radii by a factor of five, the expanded balls $B_{p_{j}}\left(r / A_{1 / 2}\right)$ again cover $S_{n}$. The former (disjointness) property guarantees that 
$k<C / r^{4}$ for some absolute constant $C$ determined by the volume of $\mathbf{P}^{2}$. The latter property guarantees that $S_{n} \subset \bigcup_{j=1}^{k} \psi_{p_{j}}\left(B_{\mathbf{0}}(r)\right)$, as desired.

To continue the proof of Theorem 1.4, we fix a point $p \in S_{n}$ and consider the map $f_{p}^{n}: B_{\mathbf{0}}\left(\delta_{1 / 2}\right) \rightarrow B_{\mathbf{0}}\left(\delta_{1 / 2}\right)$ defined as a composition

$$
f_{p}^{n}=f_{f_{+}^{n-1}(p)} \circ \ldots f_{p}
$$

of maps specified in Theorem 6.2. This map is, of course, not defined everywhere on $B_{\mathbf{0}}\left(\delta_{1 / 2}\right)$. However, if $\chi^{+}$is the largest Lyapunov exponent of $f_{+}$, then Theorem 6.2 guarantees that $f_{p}^{n}(z) \in B_{\mathbf{0}}\left(\delta_{1 / 2}\right)$ whenever $\|z\| \leq r_{n} \stackrel{\text { def }}{=}(1-\epsilon)^{n} e^{-n\left(\chi^{+}+\epsilon\right)} \delta_{1 / 2}$. Capitalizing on this observation, we set

$$
\mathcal{B}_{n, p}=\psi_{p}\left(B_{\mathbf{0}}\left(r_{n} / 2 K A_{1 / 2}\right)\right)
$$

and try to estimate $\mu\left(\mathcal{B}_{n, p}\right)$.

Lemma 7.4. There exists a constant $C$ independent of $p$ and $n$ and a local potential $g^{+}$for $\mu^{+}$on $B_{p}\left(r_{n} / A_{1 / 2}\right)$ such that $\left\|g^{+}\right\|_{\infty}<C / d^{n}$.

Proof. It follows from Theorem 6.2 that $B_{p}\left(r_{n} / A_{1 / 2}\right) \subset \psi_{p}\left(B_{\mathbf{0}}\left(r_{n}\right)\right)$. Hence from the discussion above, we have that $f_{+}^{n}\left(B_{p}\left(r_{n} / A_{1 / 2}\right)\right) \subset \psi_{f_{+}^{n}(p)}\left(B_{\mathbf{0}}\left(\delta_{1 / 2}\right)\right)$. Moreover, $f_{+}^{n}(p) \in S_{n}$ by definition. Thus we can use the potentials given by Lemma 7.2 to define a potential $g_{f_{+}^{n}(p)}^{+} \circ f_{+}^{n}$ for $f_{+}^{n *} \mu^{+}=d^{n} \mu^{+}$on $B_{p}\left(r_{n} / A_{1 / 2}\right)$. This potential is uniformly bounded above, independent of $p$ and $n$, so we can divide by $d^{n}$ to obtain the desired potential $g^{+}$for $\mu^{+}$.

Lemma 7.5. Let $\Theta$ be the Fubini-Study Kähler form on $\mathbf{P}^{2}$. There is a constant $C$ such that for every $p \in \mathbf{P}^{2}$ and $r>0$,

$$
\frac{1}{r^{2}} \int_{B_{p}(r)} \Theta \wedge \mu^{-}<C .
$$

Proof. If we work in affine coordinates centered at $p \in \mathbf{P}^{2}$ and replace the FubiniStudy Kähler form with the Euclidean Kähler form, then the left side of the desired inequality is an increasing function of $r$ (Fact 3.5). Moreover, the Euclidean and Fubini-Study Kähler forms are strongly positive forms that are comparable near the origin, so if $r_{0}>0$ is small enough, there is a constant $C=C\left(r_{0}\right)$ independent of $p$ such that for all $r<r_{0}$.

$$
\frac{1}{r^{2}} \int_{B_{p}(r)} \Theta \wedge \mu^{-} \leq \frac{C}{r_{0}^{2}} \int_{B_{p}\left(r_{0}\right)} \Theta \wedge \mu^{-} .
$$

On the other hand, we have the trivial bound

$$
\frac{1}{r^{2}} \int_{B_{p}(r)} \Theta \wedge \mu^{-}<\frac{1}{r_{0}^{2}} \int_{\mathbf{P}^{2}} \Theta \wedge \mu^{-}
$$


for all $r \geq r_{0}$.

Let $\eta_{n}: B_{0}\left(r_{n} / A\right) \rightarrow[0,1]$ be a sequence of smooth compactly supported functions identically equal to one on $B_{0}\left(r_{n} / 2 A_{1 / 2}\right)$. From the definition of $\mathcal{B}_{n, p}$ and Theorem 6.2 we have that $\eta_{n} \equiv 1$ on $\mathcal{B}_{n, p}$. Clearly, we can arrange that $d d^{c} \eta_{n} \leq C \Theta / r_{n}^{2}$. Let $g^{+}$be the local potential given by Lemma 7.4. We estimate

$$
\begin{aligned}
\mu\left(\mathcal{B}_{n, p}\right) & =\int_{\mathcal{B}_{n, p}} \mu^{+} \wedge \mu^{-} \leq \int_{\mathbf{P}^{2}} \eta_{n} d d^{c} g^{+} \wedge \mu^{-} \\
& =\int_{\mathbf{P}^{2}} g^{+} d d^{c} \eta_{n} \wedge \mu^{-} \leq \frac{C\left\|g^{+}\right\|_{\infty}}{r_{n}^{2}} \int_{B_{p}\left(r_{n} / A_{1 / 2}\right)} \Theta \wedge \mu^{-} \\
& \leq C / d^{n},
\end{aligned}
$$

where the constant $C$ is independent of $p \in S_{n}$ and $n$. The second equality is just the definition of wedge product of positive closed $(1,1)$ currents. The last inequality follows from the previous two lemmas.

To complete the proof of Theorem 1.4, we observe that $\mathcal{B}_{n, p} \supset B_{p}\left(r_{n} / 2 K A_{1 / 2}^{2}\right)$. Therefore we can apply Lemma 7.3 to choose points $p_{1}, \ldots, p_{k} \in \Lambda_{n}^{\prime}$ with $k<C / r_{n}^{4}$ such that

$$
\mu\left(\bigcup_{j=1}^{k} \mathcal{B}_{n, p_{j}}\right)>\frac{1}{8},
$$

where $C_{1}$ and $C_{2}$ are independent of $n$. From these last equations and our upper bound for $\mu\left(\mathcal{B}_{n, p}\right)$, we conclude that

$$
\frac{1}{8}<\sum_{j} \mu\left(\mathcal{B}_{n, p_{j}}\right) \leq \frac{C}{r_{n}^{4} d^{n}}
$$

for every $n$. Letting $n \rightarrow \infty$ and expanding $r_{n}$ gives

$$
\chi^{+} \geq \frac{\log d}{4(1-\epsilon)}-\epsilon .
$$

Since $\epsilon>0$ is arbitrary, Theorem 1.4 is proved.

\section{Periodic points}

If $\mu\left(\mathcal{I}^{+}\right)=\mu\left(\mathcal{I}^{-}\right)=0$, then it follows from Theorem 1.4 that $\mu$ has one positive and one negative Lyapunov exponent. Under these conditions, we will now show that saddle periodic points are dense in $\operatorname{supp} \mu$, proving Theorem 1.5. We reuse the notation $\epsilon, \Lambda, \Lambda_{t}, \delta_{t}, A_{t}$ from the previous section.

The first step of the proof consists in showing that any point in $\operatorname{supp} \mu$ can be approximated by nearly periodic regular points. 
Lemma 8.1. Given $p \in \operatorname{supp} \mu$ and $\epsilon_{1}>0$, there exists $t>0$ such that for any $\epsilon_{2}>0$, there exists $q \in \Lambda_{t}$ and $n \in \mathbf{N}$ such that

- $\operatorname{dist}(p, q)<\epsilon_{1}$.

- $f_{+}^{n}(q) \in \psi_{q}\left(B_{0}\left(\epsilon_{2}\right)\right) \cap \Lambda_{t}$.

Proof. If $t$ is small enough, the set $B_{p}\left(\epsilon_{1} / 2\right) \cap \Lambda_{t}$ has positive measure. That is, there exists $p^{\prime} \in \Lambda_{t}$ such that $B_{p^{\prime}}(r) \cap \Lambda_{t}$ has positive $\mu$ mass and is contained in $B_{p}\left(\epsilon_{1} / 2\right)$ for all $r>0$ small enough. Since $\mu$ is mixing, we can for any such $r$ find $n \in \mathbf{N}$ and $q \in B_{p^{\prime}}(r) \cap \Lambda_{t}$ such that $f_{+}^{n}(q) \in B_{p^{\prime}}(r) \cap \Lambda_{t}$ as well. Hence if $r$ satisfies $2 A_{t} r<\delta_{t}$, we have $f_{+}^{n}(q) \in B_{q}(2 r) \subset \psi_{q}\left(B_{0}(2 A(q) r)\right)$. Taking $r=\min \left\{\epsilon_{1} / 4, \epsilon_{2} / 2, \delta_{t} / 2 A_{t}\right\}$ therefore finishes the proof.

Now apply this lemma with $\epsilon_{2}<<\epsilon_{1}, \epsilon$. We will complete the proof of Theorem 1.5 by exhibiting a saddle periodic point of period $n$ whose orbit intersects $\psi_{q}\left(B_{0}\left(C \epsilon_{2}\right)\right)$. The approach is similar to the proof of the closing lemma for hyperbolic maps (Theorem 6.4.15) given in $[\mathrm{KH}]$, but there is an extra complication due to the fact that the Lyapunov charts can degenerate along the orbit of $q$. In particular, our method would not suffice to prove a general closing lemma for $f_{+}$; it is important that the pseudo-orbit $q, f_{+}(q), f_{+}^{2}(q), \ldots, f_{+}^{n-1}(q), q$ be an actual orbit except at the last step.

For each $j=1, \ldots, n-1$, we set $f_{j}=f_{f_{+}^{j-1}(q)}$, wherever the righthand side is defined-in particular on $U_{j}=B_{0}\left(e^{-\left(\chi^{+}+\epsilon\right)} \delta\left(f_{+}^{j-1}(q)\right)\right)$. Similarly, we take

$$
f_{n}=\psi_{q}^{-1} \circ f_{+} \circ \psi_{f_{+}^{n-1}(q)}=\psi_{q}^{-1} \circ \psi_{f_{+}^{n}(q)} \circ f_{f_{+}^{n-1}(q)},
$$

which, for $\epsilon_{2}$ small enough, is defined on an open subset of $\mathbf{C}^{2}$ containing $U_{n}=$ $B_{0}\left(e^{-\left(\chi^{+}+2 \epsilon\right)} \delta\left(f_{+}^{j-1}(q)\right)\right)$. We have for $0 \leq j \leq n$ that

$$
\left\|f_{j}-D_{0} f_{j}\right\|_{C^{1}}<2 \epsilon
$$

Additionally, if $j<n$ then $f_{j}(\mathbf{0})=\mathbf{0}$, and in the remaining case it is at least true that $\left\|f_{n}(\mathbf{0})\right\|<\epsilon_{2}$.

If $U=U_{1} \times \cdots \times U_{n}$, then fixed points of the map

$$
F\left(x_{1}, \ldots, x_{n}\right) \stackrel{\text { def }}{=}\left(f_{n}\left(x_{n}\right), f_{1}\left(x_{1}\right), \ldots, f_{n-1}\left(x_{n-1}\right)\right)
$$

from $U$ into $\mathbf{C}^{2 n}$ correspond to periodic orbits $\psi_{q}\left(x_{1}\right), \ldots, \psi_{f^{n-1}(q)}\left(x_{n}\right)$ of $f_{+}$. To find a fixed point, we write $F=D_{0} F+E$, where $E(\mathbf{0})=\left(f_{n}(\mathbf{0}), \mathbf{0}, \ldots, \mathbf{0}\right)$ and in the product norm (this is essential, since we have no control on the size of $n$ ) on $\mathbf{C}^{2 n}=\left(\mathbf{C}^{2}\right)^{n}$, we have $\|E\|_{C^{1}}<2 \epsilon$. Moreover, the linear operator, $D_{\mathbf{0}} F$ is a shifted product of the $D_{0} f_{j}$ 's, so $\left\|\left(D_{0} F-I\right)^{-1}\right\|$ is bounded above by a constant depending only on the minimal distance from the Lyapunov exponents of $\mu$ to zero.

A point is fixed by $F$ if and only if it is fixed by $S \stackrel{\text { def }}{=}\left(D F_{0}-I\right)^{-1} \circ E$. Moreover, the above observations show that $S$ contracts the product metric by a factor of $2 C \epsilon$, where again, $C$ depends only on the Lyapunov exponents of 
$\mu$. We claim that $S(U) \subset U$ for $\epsilon$ and $\epsilon_{2}$ taken small enough to invoke the (proof of the) contraction mapping theorem. To see this, let $S_{j}$ denote the $j$ th component of $S$. It is possible that the radius of $U_{j}$ might decay as $j$ increases from 1 to $n$. Nevertheless, slow variation of $\delta$ ensures that the radius of $U_{j+1}$ is at least $(1-\epsilon)$ times that of $U_{j}$. Therefore since $x_{j} \in U_{j}$ for $j<n$, we see that $\left\|S_{j+1}\left(x_{j}\right)\right\|=\left\|S_{j+1}\left(x_{j}\right)-S_{j+1}(\mathbf{0})\right\|=2 C \epsilon\left\|x_{j}\right\|$, so that $S_{j+1}\left(x_{j}\right) \in U_{j+1}$. Moreover, continuity of $\delta$ on $\Lambda_{t}$ allows us to assume by shrinking $\epsilon_{2}$ that the radii of $U_{n}$ and $U_{1}$ are nearly equal. Hence $x_{n} \in U_{n}$ implies that $\left\|f_{n}\left(x_{n}\right)\right\| \leq \epsilon_{2}+2 C \epsilon\left\|x_{n}\right\|$ so that $S_{1}\left(x_{n}\right) \in U_{1}$, too. Our claim is therefore justified.

Thus

$$
\left\|S^{n}(\mathbf{0})\right\| \leq \sum_{j=1}^{n}\left\|S^{j}(\mathbf{0})-S^{j-1}(\mathbf{0})\right\| \leq\|S(\mathbf{0})\| \sum_{j=0}^{n-1}(2 C \epsilon)^{j} \leq 2 \epsilon_{2}
$$

for $\epsilon$ small enough. In particular, $X=\lim _{n \rightarrow \infty} S^{n}(\mathbf{0})$ exists, is a fixed point of $F$, and lies within distance $2 \epsilon_{2}$ of the origin. The image $q^{\prime}=\psi_{1}\left(x_{1}\right)$ of the first coordinate of $X$ is a periodic point of period (dividing) $n$ close to $q$. Since its orbit is contained in the Lyapunov charts about $q, \ldots, f^{n-1}(q)$, we see easily that the largest and smallest eigenvalues of $D_{q^{\prime}} f^{n}$ have magnitudes approximately $e^{n \chi^{+}}$ and $e^{n \chi^{-}}$, respectively, so that $q^{\prime}$ is also a saddle point. This concludes the proof of Theorem 1.5.

\section{References}

[BS] E. Bedford and J. Smillie, Polynomial diffeomorphisms of $\mathbf{C}^{2}$, III: ergodicity, exponents and entropy of the equilibrium measure, Math. Ann. 294 (1992), 395-420.

[BT] E. Bedford and B. A. Taylor, The Dirichlet problem for a complex Monge-Ampere operator, Inventiones Math. 37 (1976), 1-44.

[Bri] J.-Y. Briend, Exposants de Liapounoff et Points Périodiques d'Endomorphismes Holomorphes de $\mathbf{C P}^{\mathbf{k}}$, l'université Paul Sabatier-Toulouse III, 1997.

[BD] J.-Y. Briend and J. Duval, Exposants de Liapounoff et distribution des points périodiques d'un endomorphisme de $\mathbf{C P}^{k}$, Acta Math. 182 (1999), 143-157.

[Dem] J.-P. Demailly, Monge-Ampère operators, Lelong numbers, and intersection theory, in Complex Analysis and Geometry (V. Ancona and A. Silva, editors), 115-193. Plenum Press, 1993.

[Dil1] J. Diller, Dynamics of birational maps of $\mathbf{P}^{2}$, Indiana Univ. Math. J. 45 (1996), 721-772.

[Dil2] J. Diller, Birational maps, positive currents, and dynamics, Michigan Math. J. 46 (1999), 361-375.

[Fav] C. Favre, Points périodiques d'applications birationnelles de $\mathbf{P}^{2}$, Ann. Inst. Fourier (Grenoble), 48 (1998), 999-1023.

[FS1] J. E. Fornæss and N. Sibony, Complex dynamics in higher dimension, II, in Modern Methods in Complex Dynamics, volume 137 of Ann. of Math. Stud., 135-182. Princeton Univ. Press, 1995

[FS2] J. E. Fornæss and N. Sibony, Oka's inequality for currents and applications, Math. Ann. 301 (1996), 399-419.

$[\mathrm{KH}]$ A. Katok and B. Hasselblatt, Introduction to the Modern Theory of Dynamical Systems, Cambridge University Press, 1995. 
[Kli] M. Klimek, Pluripotential Theory, Oxford University Press, 1991.

[Sib] N. Sibony, Dynamique des applications rationnelles de $\mathbf{P}^{k}$, in Dynamique et géométrie complexes (Lyon, 1997), ix-x, xi-xii, 97-185. Soc. Math. France, Paris, 1999.

[Ste] E. M. Stein, Singular Integrals and Differentiability Properties of Functions, Princeton University Press, 1970.

Jeffrey Diller

Department of Mathematics

University of Notre Dame

Notre Dame

IN 46556

USA

e-mail: diller.1@nd.edu

(Received: January 3, 2001) 